\title{
Factores predisponentes de abandono temprano en estudiantes de Medicina Predisposing factors of student drop-out early in the career of Medicine
}

\author{
Rony Ríos*, Rebeca Peña, Miguel Aguilar
}

Facultad de Ciencias Médicas

Universidad de San Carlos de Guatemala

Autor al que se dirige correspondencia: rios.rony@usac.edu.gt

Recibido: 30 de marzo de 2016 / Aceptado: 11 de septiembre de 2016

\section{Resumen}

$\mathrm{E}$ 1 abandono estudiantil temprano es un problema frecuente, generalmente provocado por mala orientación vocacional y aspectos motivacionales. Para determinar los factores que predisponen al mismo, se realizó un estudio de casos $(\mathrm{N}=391)$ y controles $(\mathrm{N}=782)$ incluyendo a los primocursantes de las cohortes 2010, 2012 y 2013 inscritos en primer año de la carrera de medicina de la Facultad de Ciencias Médicas de la Universidad de San Carlos de Guatemala (Usac), que desertaron al año siguiente de su primera asignación. Se consideraron factores personales, socioeconómicos y académicos, para determinar si existía relación estadísticamente significativa con el abandono estudiantil. Los resultados obtenidos indican que el nivel educativo del padre (OR 1.4 IC 1.07 - 1.83), la religión (OR 1.65 IC 1.03 - 2.66) y el hecho de efectuar un trabajo remunerado simultáneo con los estudios (OR 1.49 IC 1.03 - 2.14), además del resultado final en los cursos de primer año de Química (OR 7.54 IC 5.56 - 10.24), Física (OR 6.74 IC 4.88 - 9.32), Biología (OR 6.86 IC 5.09 - 9.23) y Psicología (OR 5.92 IC $4.38-8.01)$, son factores que predisponen al abandono estudiantil $(\mathrm{p}<0.05)$. Se sugieren acciones para disminuir la repitencia en estos cursos y con ello evitar el abandono temprano en la Facultad de Ciencias Médicas de la Usac, así como dar un seguimiento estrecho a estudiantes que se inscriban en los próximos años y que presenten los factores de riesgo mencionados para evitar que abandonen.

Palabras clave: Estudiante universitario, alumno desertor, fracaso escolar, empleo del estudiante, educación de los padres

\section{Abstract}

S tudent early drop out is a common problem often caused by poor career counseling and motivational aspects. To determine the factors or aspects that predispose to it, is carried out a study of cases $(\mathrm{N}=391)$ and controls $(\mathrm{N}=782)$ ratio of 1:2 including freshmen of the cohorts 2010, 2012 and 2013, of the career of medicine of the Faculty of Medical Sciences of the University of San Carlos of Guatemala (Usac), who had deserted from the Faculty the following year for his first assignment. It is considered demographic, academics, family, labor, health and motivational factors, studying the most important in defection. The results indicate that the educational level of the parents (OR 1.4 CI $1.07-1.83$ ), religion (OR 1.65 CI $1.03-2.66$ ) and the fact to perform a paid work simultaneously with the studies (OR 1.49 CI $1.03-2.14$ ), besides the final results in the courses of Chemistry (OR 7.54 CI 5.56 - 10.24), Physics (OR 6.74 CI 4.88 - 9.32), Biology (OR 6.86 CI 5.09 - 9.23) and Psychology (OR 5.92 CI $4.38-8.01)$, are factors that predispose the student to dropout from school $(\mathrm{p}<0.05)$. Actions had been taken into consideration to prevent the repetition of these academic subjects and thereby avoid early abandonment from the School Medicine and to keep closely monitoring of the students who will enroll in the coming years and that show or present the above risk factors in order to prevent the students from leaving. 


\section{Introducción}

El abandono y la repitencia estudiantil son dos problemas importantes en las instituciones universitarias que inciden en una disminución significativa de su eficiencia terminal. Algunas de las consecuencias de ello son: la larga duración de los estudios de pregrado, mayores gastos incurridos por los estudiantes, gastos extras en el caso de las instituciones públicas de educación superior, retraso en la entrada al mercado laboral lo que genera costos sociales y económicos (Castaño, Gallón, Gómez, \& Vásquez, 2006). Asociado a ello existe un creciente surgimiento de universidades privadas que adaptan las carreras a las necesidades empresariales, lo que cuestiona, tanto al interior de las universidades, como al exterior por parte de los medios de comunicación y la población en general, la eficacia de las universidades públicas. (Cabrera, Bethencourt, Alvarez, \& González, 2006).

Aunque existen muchas teorías para explicar la decisión de un estudiante de abandonar sus estudios universitarios, los modelos teóricos más aceptados son el modelo de integración estudiantil de Tinto y el modelo de desgaste estudiantil de Bean. Tinto argumenta que la adaptación social y académica del estudiante a la universidad determinan la decisión de permanecer o no en sus estudios. Bean, con una visión más amplia, considera que para tomar tal medida, existen otros factores ajenos a la universidad: académicos, personales y psicosociales (Castaño et al., 2006)

En la deserción temprana, entendida como el abandono de la carrera universitaria por parte del estudiante en los primeros semestres de la carrera, los factores reportados que más inciden son la orientación vocacional, la adaptación a la vida universitaria y el aspecto motivacional (Ríos de los \& Canales, 2007; Sánchez, Navarro, \& García, 2009)

La matrícula estudiantil en educación superior en Guatemala, tanto en el sector público como el privado, ha tenido un crecimiento de $210 \%$ entre 1994 y 2008. De manera individual, la Universidad de San Carlos de Guatemala atiende la mayor cantidad de estudiantes en el país que actualmente representa el $70 \%$ del total de matriculados. El número de estudiantes inscritos en el 2014 en todo el país alcanzó un total de 195,000. (Cárdenas, 2014). En la Facultad de Ciencias Médicas, el número de primocursantes de primer año ha variado desde que se implementaron las pruebas de ubicación para el ingreso, en el último quinquenio, dicha cifra ha oscilado entre 700 a 950 estudiantes (Ríos,
2010). Aunque los índices de repitencia y deserción han disminuido comparándolos con los de años anteriores, aún permanecen elevados y son motivo de preocupación para las autoridades facultativas.

Este estudio aborda la relación entre las características previas del sujeto y su éxito en los primeros años en la universidad, determinando los factores predisponentes al abandono en estudiantes de primer año de la carrera de medicina en la Facultad de Ciencias Médicas de la Universidad de San Carlos de Guatemala (Usac) en las cohortes 2010, 2012 y 2013. Se considera como abandono a aquel estudiante que luego de haber estudiado un año en la Facultad, no se asigna cursos al año siguiente. No se estudió la cohorte 2011 por no contarse con los datos en la oficina de registro.

Como antecedente en Guatemala para la carrera de Medicina, se reporta que hace más de 20 años Cambranes determinó un $10 \%$ de deserción y concluyó que "el alto índice de repitencia, el bajo rendimiento académico y la personalidad deficientes son las causas principales de deserción en estudiantes de 2do. año de la carrera" (1991). Otro estudio realizado por Calderón (2005) hace 10 años, encontró que la tasa de deserción fue del $47 \%$ y que las razones para desertar, desde el ámbito del estudiante fueron: el factor motivacional, el desempleo y las limitaciones académicas, el rendimiento relacionado con limitaciones en el aprendizaje y la presión familiar. Este estudio tiene la limitante importante del escaso número de la muestra, apenas 6 estudiantes en total.

Por investigaciones previas, se sabe que las tasas de deserción son menores en las facultades donde existe un mayor grado de selectividad y donde el número de aspirantes supera al de plazas disponibles. Aunque en la Usac no se tiene un número límite de plazas, específicamente para la Facultad de Ciencias Médicas, durante los últimos 5 años ha habido entre 3000 a 4000 solicitudes anuales. Sin embargo, el índice de abandono estudiantil temprano permanece elevado, siendo del 15.6\% en el año 2012 (Ríos \& Pineda, 2014).

Considerando que no existen suficientes antecedentes acerca del fenómeno de abandono estudiantil en la Facultad de Ciencias Médicas de la Usac, se decidió estudiar algunos factores predisponentes en el estudiante, abarcando su ámbito personal, socioeconómico y académico. Se tomaron los datos del cuestionario de asignación de pruebas específicas previo a ingresar a la Universidad y se buscó la asociación con el hecho de abandonar los estudios luego de cursado el primer año. 


\section{Materiales y métodos}

Se realizó un estudio observacional analítico de casos y controles, analizando los factores anotados al ingreso por los estudiantes de la Facultad para identificar los que se relacionaron con el abandono estudiantil. Para el efecto, se incluyeron a 391 estudiantes correspondientes a todos los desertores de 1er. año de la Facultad de Ciencias Médicas en las cohortes 2010 ( $\mathrm{n}=$ 67), $2012(n=133)$ y $2013(n=191)$. Los valores del grupo de desertores se contrastaron con los obtenidos del grupo control. La relación de controles por cada caso fue de 2:1 para aumentar la potencia estadística. Los controles se obtuvieron de estudiantes de las mismas cohortes pero que no abandonaron y se escogieron por medio de un muestreo probabilístico sin reemplazo, en forma aleatoria.

Las variables predictoras se clasificaron en: personales (edad, sexo, estado civil, nacionalidad, etnia, número de hermanos, puesto que ocupa entre ellos, razones personales para estudiar medicina, pertenencia a grupos culturales, religiosos, práctica de algún instrumento musical, práctica de algún deporte); académicas (título de enseñanza media, tipo de establecimiento, resultados de pruebas de ingreso, notas finales de cursos de 1er. año de la carrera de medicina); socioeconómicas (trabajo remunerado, dependencia económica situación familiar, escolaridad de los padres, ingreso económico familiar mensual).

Todas las variables enmarcadas dentro de esta caracterización fueron analizadas con el programa estadístico SPSS 17.0.1, realizando un análisis discriminante llevado a cabo con pruebas de independencia por el método de de Ji-cuadrado para establecer asociación a un nivel de significancia de 0.05 y posteriormente se calcularon las razones de oportunidades u odds ratio, por sus siglas en inglés (OR) para establecer la fuerza de dicha asociación. Con el análisis de regresión logística y debido a las diferencias en los valores de $p$, se construyeron dos modelos predictivos: uno con las variables académicas y el otro con las variables no académicas.

\section{Resultados}

Las características generales de los grupos estudiados, 391 estudiantes catalogados como "desertores", que constituyeron los casos (33\%) y 782 estudiantes que constituyeron los controles (67\%), se indican en la tabla 1.
Del total de los casos, la mitad prácticamente $(\mathrm{n}=$ 191) pertenecían a la cohorte 2013 y el menor número $(n=67)$ a la cohorte 2010; el resto pertenecían a la cohorte 2012. Los controles fueron incluidos en la misma proporción de la de los casos, para cada cohorte.

En la tabla se determina, en cuanto al sexo, que hay un leve predominio del sexo masculino, con un $35 \%$ comparado con el $32 \%$ de mujeres a pesar que en el grupo en general hay más mujeres que hombres. La edad promedio en ambos grupos es de 19 años. Con relación a la escolaridad del padre y al trabajo remunerado por parte de los estudiantes, existe una diferencia entre los casos y los controles. De la primera variable mencionada se evidencia que el $72 \%$ de los estudiantes que abandonaron tienen a padres no universitarios, comparados con el $65 \%$ de los no lo hicieron. De la segunda variable mencionada, se determina que el $15 \%$ de los desertores tenían un trabajo remunerado al momento de llenar el cuestionario, comparados con el $11 \%$ de los no desertores.

Para el resto de variables generales que se consignan en la tabla 1 , los valores son bastante homogéneos: estado civil, grupo étnico, procedencia, tipo de establecimiento donde cursaron sus estudios secundarios y título o diploma de enseñanza media.

Con los resultados obtenidos luego del análisis de regresión logística consignados en la tabla 2 , se construyeron dos modelos predictivos. El primero relaciona las variables académicas y la ecuación es:

$\mathrm{P}(\mathrm{Y}=1)=\frac{1}{1+\exp ^{(-0.698+0.711 \times 1+0.758 \times 2+0.695 \times 3+0.882 \times 4)}}$

Los resultados obtenidos luego del análisis de regresión logística evidencian que las variables académicas predominan sobre las variables no académicas A través del modelo para variables académicas se estima que la probabilidad de abandonar es del $67 \%$ en un estudiante que repruebe las unidades didácticas de Química, Física, Biología y Psicología, en tanto que si el estudiante aprueba dichas unidades, la probabilidad de que abandone la Facultad es $8.72 \%$.

El modelo creado para variables no académicas predice una probabilidad de abandono del $52 \%$ cuando el estudiante presenta las siguientes condiciones: No profesar la religión católica o evangélica, tener un trabajo remunerado y que el padre de familia no sea universitario. Para el resto de variables incluidas en el estudio, no se encuentra asociación de ellas con el hecho de abandonar los estudios. 
Tabla 1

Características generales de 1173 estudiantes de 1er. año de la Facultad de Ciencias Médicas, Usac, cohortes 2010, 2012 y 2013

\begin{tabular}{|c|c|c|c|c|}
\hline \multirow[t]{2}{*}{ Características } & \multicolumn{2}{|c|}{ Casos $\mathrm{N}=391$} & \multicolumn{2}{|c|}{ Controles $\mathrm{N}=782$} \\
\hline & Frecuencia & Porcentaje & Frecuencia & Porcentaje \\
\hline \multicolumn{5}{|l|}{ Sexo } \\
\hline Masculino & 201 & 51.41 & 377 & 48.21 \\
\hline Femenino & 190 & 48.59 & 405 & 51.79 \\
\hline Edad en años & 19.24 & 1.22 & 18.97 & 2.13 \\
\hline \multicolumn{5}{|l|}{ Estado civil } \\
\hline Soltero & 369 & 97.11 & 739 & 98.14 \\
\hline Casado & 11 & 2.89 & 14 & 1.86 \\
\hline \multicolumn{5}{|l|}{ Grupo étnico } \\
\hline Ladino & 352 & 94.37 & 692 & 93.77 \\
\hline Maya & 21 & 5.63 & 45 & 6.23 \\
\hline \multicolumn{5}{|l|}{ Lugar de procedencia } \\
\hline Ciudad capital & 311 & 81.41 & 630 & 82.79 \\
\hline Departamentos & 71 & 18.59 & 131 & 17.21 \\
\hline \multicolumn{5}{|l|}{ Tipo de establecimiento } \\
\hline Público & 20 & 5.29 & 48 & 6.38 \\
\hline Privado & 358 & 94.71 & 704 & 93.62 \\
\hline \multicolumn{5}{|l|}{ Cohorte } \\
\hline 2010 & 67 & 17.14 & 134 & 17.14 \\
\hline 2012 & 133 & 34.02 & 266 & 34.02 \\
\hline 2013 & 191 & 48.85 & 382 & 48.85 \\
\hline \multicolumn{5}{|l|}{ Escolaridad del padre } \\
\hline No universitario & 265 & 70.48 & 469 & 63.04 \\
\hline Universitario & 111 & 29.52 & 275 & 36.96 \\
\hline \multicolumn{5}{|l|}{ Trabajo remunerado } \\
\hline Sí & 57 & 15.16 & 81 & 10.73 \\
\hline No & 319 & 84.84 & 674 & 89.27 \\
\hline \multicolumn{5}{|l|}{ Religión } \\
\hline No cristiana & 33 & 8.68 & 41 & 5.44 \\
\hline Cristiana & 347 & 91.32 & 713 & 94.56 \\
\hline \multicolumn{5}{|l|}{ Escolaridad de la madre } \\
\hline No universitaria & 280 & 74.67 & 526 & 70.79 \\
\hline Universitaria & 95 & 25.33 & 217 & 29.91 \\
\hline \multicolumn{5}{|l|}{ Hogar integrado } \\
\hline Sí & 75 & 29.18 & 224 & 29.13 \\
\hline No & 182 & 70.82 & 545 & 70.87 \\
\hline \multicolumn{5}{|l|}{ Pruebas de ingreso } \\
\hline Promedio de básico y diversificado & $\square 78.57$ & $\sigma \quad 6.08$ & $\square 81.54$ & $\sigma \quad 6.71$ \\
\hline Promedio de conocimientos básicos & $\square 64.92$ & $\sigma 7.53$ & $\square 67.07$ & $\sigma 8.22$ \\
\hline Habilidad verbal & $\square 57.93$ & $\sigma 22.02$ & $\square 57.49$ & $\sigma 21.91$ \\
\hline Razonamiento numérico & $\square 63.13$ & $\sigma 18.96$ & $\square 63.49$ & $\sigma 18.86$ \\
\hline Aptitud académica & $\square 41.15$ & $\sigma 10.38$ & $\square 41.11$ & $\sigma 10.34$ \\
\hline
\end{tabular}


Tabla 2

Variables significativas para abandonar los estudios, 1173 estudiantes de ler. año de la Facultad de Ciencias Médicas, Usac, cohortes 2010, 2012 y 2013

\begin{tabular}{lcccc}
\hline \multicolumn{1}{c}{ Variable } & Ji cuadrado & Odds ratio or & $\begin{array}{c}\text { Intervalos de } \\
\text { confianza }\end{array}$ & Valor $\mathrm{p}$ \\
\hline U.D.* de Química & 193.76 & 7.54 & $5.56-10.24$ & 0 \\
U.D. de Biología & 182.90 & 6.86 & $5.09-9.23$ & 0 \\
U.D. de Física & 157.14 & 6.74 & $4.88-9.32$ & 0 \\
U.D. de Psicología & 150.53 & 5.92 & $4.38-8.01$ & 0 \\
Religión & 4.36 & 1.65 & $1.03-2.66$ & 0.03 \\
Padre no universitario & 6.12 & 1.4 & $1.07-1.83$ & 0.013 \\
Trabaja & 4.64 & 1.49 & $1.03-2.14$ & 0.039
\end{tabular}

* U.D. Unidad Didáctica en primer año.

La ecuación del modelo de variables no académicas, es el siguiente:

$$
\mathrm{P}(\mathrm{Y}=1)=\frac{1}{1+\exp ^{(0.230+0.429 \times 1+0.322 \times 2-0.337 \times 3)}}
$$

Debido a que no se contaba con la totalidad de los datos en algunas variables, se hicieron análisis parciales con los que se recuperaron de forma incompleta. Estos hallazgos evidencian que: el puesto que ocupa el estudiante entre los hermanos, el dominio de idiomas extranjeros (uno o más), el nivel socioeconómico del estudiante, la practica regular de algún deporte y el tener un hogar integrado no se asocian con el abandono estudiantil. Las únicas variables que tuvieron una asociación estadísticamente significativa $(\mathrm{p}<0.05)$ son: la escolaridad de la madre y el hecho de no interpretar algún instrumento musical. En cuanto a la escolaridad de la madre, existe de 1.80 a 3.65 veces la posibilidad de que un estudiante que no tenga madre profesional universitaria, abandone sus estudios en la Facultad. Con el otro factor, las posibilidades de abandonar oscilan entre 1.02 a 1.91 veces. Esto significa que el interpretar un instrumento musical, es un factor protector que previene el abandono estudiantil. (Tabla 3)

Tabla 3

Variables significativas para abandonar los estudios en un grupo de estudiantes de ler año de la Facultad de Ciencias Médicas, USAC. Cohortes 2010, 2012 y 2013

\begin{tabular}{lcccc}
\hline \multicolumn{1}{c}{ Variable } & Ji cuadrado & Odds ratio & $\begin{array}{c}\text { Intervalos de } \\
\text { confianza }\end{array}$ & Valor $\mathrm{p}$ \\
\hline Dominio de idiomas extranjeros & 0.31 & 1.080 & $0.68-1.72$ & 0.590 \\
Practicar deportes & 0.17 & 1.070 & $0.77-1.50$ & 0.680 \\
No interpretar un instrumento musical & 4.35 & 1.400 & $1.02-1.91$ & 0.040 \\
Hogar Integrado & 0.64 & 1.004 & $0.72-1.40$ & 0.970 \\
Madre no universitaria & 28.81 & 2.570 & $1.80-3.65$ & 0.000 \\
\hline
\end{tabular}




\section{Discusión}

La mayoría de investigadores coinciden en que el rendimiento académico inadecuado es una causa importante de abandono. Dicho rendimiento puede verse influido en la carrera de Medicina por: duración excesiva del plan de estudios, inadecuada formación pedagógica por los docentes rigidez en horarios de clases y deficiencia en conocimientos previos (Boado, 2007; López, Marín, \& García, 2012). La repitencia y la deserción son fenómenos que en muchos casos están vinculados, ya que la investigación demuestra que la repitencia reiterada conduce, por lo general, al abandono de los estudios (Centro Interuniversitario de Desarrollo, \& Instituto de Educación Superior para América Latina y el Caribe, 2006).

Con relación al modelo académico, se ha demostrado que, en la Facultad de Ciencias Médicas, las unidades didácticas de Química, Física, Estadística y Biología son los que presentan los índices de repitencia más altos (Ríos, 2010). Se destaca el hecho que no se encuentra la unidad de Estadística asociada al abandono estudiantil y que se asocia el curso Psicología a dicho evento. La reflexión que se puede hacer de ello tiene 2 variantes: o se le aumentó el nivel de dificultad al curso de Psicología o el hecho de reprobar un curso considerado por los estudiantes como relativamente fácil, los desmotiva o desmoraliza e influye en su decisión de abandonar los estudios de Medicina. Se habla de facilidad relativa de ese curso ya que en la última década los índices de repitencia han oscilado entre el 10 y el 48\%, comparándolo con los índices de las cursos indicados al principio de este párrafo, en los cuales dicha variación ha ido del 30 hasta el $85 \%$ (Ríos, 2010).

Estudios realizados en la Facultad de Ciencias Médicas de la Usac evidencian que la probabilidad de éxito en estudiantes repitentes, disminuye con relación a las veces que repiten el mismo grado (Soto, 2007) y que los estudiantes que logran aprobar los cursos de Biología y Química tienen mayores probabilidades de éxito que los que no lo consiguen (González, 2011).

Con relación al segundo modelo, hay 3 variables no académicas que se asocian a la deserción: padre no universitario, no profesar la religión católica o evangélica, y poseer un trabajo remunerado. En cuando al nivel de estudios de los padres, los autores difieren en su importancia ya que algunos no han encontrado evidencia que sea un factor de asociación (Vaira, Ávila, Ricardi, \& Bergesio, 2010) mientras que otros coinciden con los resultados obtenidos en el presente trabajo, en que la educación del papá si es un factor asociado a deserción (Celis, Flores, Reyes, \& Venegas, 2013; Mairata, Miró, Montaño, Palou, \& Sánchez, 2010; Vaira, Ricardi, Taborda, Arralde, \& Manni, 1997). Puede asumirse que los padres con títulos universitarios además de ser un modelo para sus hijos, pueden ser un poco más exigentes en cuanto al estudio, lo que implicaría menos probabilidades de desertar. Pero también puede pensarse que las obligaciones profesionales que tienen, limitan el tiempo para estar en su hogar y supervisar a sus hijos universitarios, lo que podría ser una limitación que podría influir en que los muchachos abandonen la universidad.

El hallazgo relativo a la religión no había sido publicado anteriormente. Se encontró en este estudio que no ser cristiano católico o evangélico es un factor predisponente para el abandono estudiantil. Bajo la variable religión se incluyen las 2 más practicadas entre los estudiantes de Medicina (Ríos, 2009) y en general, en la población guatemalteca: católicos y evangélicos. Pueda ser que los estudiantes cristianos con estas denominaciones, encuentren en su religión la fuerza para permanecer en la universidad a pesar de las vicisitudes que se presentan, aunque necesariamente se necesitan mayores estudios en este aspecto para aceptar o rechazar la hipótesis.

Finalmente con relación a la tercera variable del modelo: trabajo remunerado, existe un consenso generalizado que trabajar al mismo tiempo que se estudia es un factor importante para deserción. (Diconca, dos Santos, \& Egaña, 2011; Mairata et al., 2010; Sánchez et al., 2009). Este hallazgo en el estudio presenta una asociación débil pero resulta lógico que se relacione, ya que el trabajo quita tiempo para estudiar, implica otras preocupaciones y probablemente también sea efectuado por estudiantes con cargas familiares.

Otro hallazgo casual que puede ser fuente de futuras investigaciones es el papel de la música relacionada con el rendimiento académico. En este estudio se encuentra asociación entre la no interpretación de un instrumento musical y el abandono, lo que podría hacer pensar que dicho hábito pueda tener alguna influencia que incida en la persistencia para estudiar o fortalezca a la persona para aceptar de mejor manera situaciones adversas.

Este trabajo tiene entre otras, las limitantes de no haber evaluado los hábitos de estudio de la población y de no haber considerado las carreras que la sección de Orientación Vocacional le sugiere al estudiante como primeras opciones para ingresar a la Universidad. Otra 
limitante es no haber evaluado los factores institucionales que se relacionan con la repitencia estudiantil, la que influye en forma importante en el abandono.

Se concluye que los factores que predisponen a la deserción estudiantil en el primer año de la carrera de medicina, se agrupan en 2 aspectos:

Académicos. Incluye la no aprobación de las siguientes unidades didácticas: Química, Física, Biología y Psicología.

No académicos. Incluye el hecho de no profesar la religión católica o evangélica, el que el padre no sea universitario y que el estudiante debe efectuar un trabajo remunerado simultáneamente con sus estudios.

Los resultados obtenidos en el presente estudio, pretenden abrir las puertas hacia la planificación de nuevas estrategias de apoyo estudiantil con una visión más sistemática de la realidad política, económica y social. Se debe considerar el diseño de nuevos sistemas de prestación de servicios (Programas remediales y/o cursos de nivelación en instituciones educativas de nivel medio), programas integrales de asesoramiento y apoyo al estudio, sobre todo de entrenamiento en aprendizaje y apoyo psicológico.

Se sugiere implementar también otros programas remediales para compensar las deficiencias en la formación secundaria, otorgar más becas dirigidas, reforzar actividades de orientación vocacional en la USAC y/o de ser posible, implementarlas en el nivel medio. La Facultad de Ciencias Médicas debe implementar acciones para disminuir los índices de repitencia en los 4 cursos que aparecen como predictores. Puede implementarse también un proyecto de donación de libros básicos para la carrera, a los estudiantes de peor condición socio-económica, y/o apoyar grupos de estudio formados a iniciativa de los propios estudiantes asistidos por un docente.

\section{Agradecimientos}

Se agradece el financiamiento proporcionado por Dirección General de Investigación (Partida 4.8.63.5.72) y por la Facultad de Ciencias Médicas, ambas de la Universidad de San Carlos de Guatemala (Usac).

\section{Referencias}

Boado, M. (2007). Una aproximación a la deserción estudiantil universitaria en Uruguay. Montevideo: Organización de las Naciones Unidas para la Educación, la Ciencia y la Cultura (Unesco) -Instituto Internacional de la Unesco para la Educación Superior en América Latina y el Caribe (Iesalc).

Cabrera, L., Bethencourt, J. T., Alvarez, P., \& González, M. (2006). El problema del abandono de los estudios universitarios. Relieve, 12(2), 171-202.

Calderón, J. L. (2005). Estudio sobre la repitencia $y$ deserción en la educación superior de Guatemala. Guatemala: Organización de las Naciones Unidas para la Educación, la Ciencia y la Cultura.

Cambranes, E. (1991). Deserción estudiantil universitaria: Estudio descriptivo en estudiantes de segundo año de la carrera de médico y cirujano que desertaron en la lra. unidad o que terminaron la $1^{\text {ra. }}$ pero desertaron en el inicio de la $2^{\text {da. }}$ Unidad, durante el año de 1991. Guatemala (Tesis de licenciatura). Universidad de San Carlos de Guatemala, Guatemala.

Cárdenas, I. (Ed.). (31 de mayo de 2014). Inscritos 2014: 195 mil Estudiantes. Periódico Universidad, 237, p.7.

Castaño, E., Gallón, S., Gómez, K., \& Vásquez , J. (julio-diciembre de 2006). Análisis de los factores asociados a la deserción y graduación estudiantil universitaria. Lecturas de Economía, 65, 9-36.

Celis, R., Flores, C. L., Reyes, M. C., \& Venegas, H. (2013). Factores de riesgo de deserción presentes en alumnos repitentes de las carreras de Enfermería y Kinesiología en una universidad chilena. Ciencia y Enfermeria, 19(3), 63-71.

Ríos de los, D., \& Canales, A. (2007). Factores explicativos de la deserción universitaria. Calidad en la Educación, 26, 173-201.

Diconca, B. (Coord.), dos Santos, S., \& Egaña, A. (2011). Desvinculación estudiantil al inicio de una carrera universitaria. Uruguay: Universidad de la República Uruguay.

González, S. del R. (2011). Exploración de factores que predigan el éxito académico Facultad de Ciencias Médicas de la Universidad de San Carlos de Guatemala cohorte 2003 (Tesis de maestría). Universidad de San Carlos de Guatemala, Facultad de Humanidades, Escuela de Estudios de Postgrado, Guatemala.

López, I., Marín, G., \& García, M. E. (2012). Deserción escolar en el primer año de la carrera de Me- 
dicina. Revista de Educación Médica Superior, 26(1), 45-52.

Mairata, M. J., Miró, R., Montaño, J. J., Palou, M., \& Sánchez, R. (2010). Seguimiento de las titulaciones: El abandono de los estudios universitarios. Recuperado de http://www.uclm.es/organos/ vic_docencia/eca/foroAlmagro/XIIForoalmagro/ descargas/Documentos-Trabajo/02.UIB.pdf

Ríos, R. E. (2009). Caracterización de los estudiantes de primer año de la Facultad de Ciencias Médicas. Guatemala: Facultad de Ciencias Médicas, Unidad de Apoyo y Desarrollo Estudiantil.

Ríos, R. (2010). Utilidad de las pruebas de ingreso en la Facultad de Ciencias Médicas de la Universidad de San Carlos de Guatemala, del año 2003 al año 2008. Guatemala: Facultad de Ciencias Médicas, Unidad de Apoyo y Desarrollo Estudiantil.

Ríos, R., \& Pineda, L. (2014). Factores relacionados con deserción temprana en estudiantes de Medicina. Trabajo presentado en la IV Conferencia Latinoamericana Sobre el Abandono en la Educación Superior, Medellín.
Sánchez, G., Navarro, W., \& García, A. (mayo 2009). Factores de deserción estudiantil en la Universidad Surcolombiana. Paidea Surcolombiana, 14, 97-103.

Soto, H. (2007). Modelo predictivo del rendimiento académico en estudiantes repitentes de primer año. Facultad de Ciencias Médicas. Tesis, Guatemala.

Centro Interuniversitario de Desarrollo, \& Instituto de Educación Superior para América Latina y el Caribe. (2006). Repitencia y deserción universitaria en América Latina. Santiago, Chile: Autor

Vaira, S., Ávila, O., Ricardi, P., \& Bergesio, A. (2010). Deserción universitaria. Un caso de estudio: variables que influyen y tiempo que demanda la toma de decisión. FABICIB, 14, 107-115. doi: www.dx.doi.org/10.14409/fabicib.v14i1.856

Vaira, S., Ricardi, P., Taborda, L., Arralde, Z., \& Manni, D. (1997). Egreso y deserción universitaria: El caso de cohortes de carreras de la UNL. Perfil social. Recuperado de http://www.alfaguia.org/alfaguiav2/bin/articulos.php?idFicha $=162$ 